Website: http://revistas.lamolina.edu.pe/index.php/acu/index

(C) Universidad Nacional Agraria La Molina, Lima - Perú

\title{
Evaluación de la calidad del agua en el río Mashcón, Cajamarca, 2016
}

\author{
Assessment of water quality in the Mashcón River, Cajamarca, 2016
}

\author{
Pedro Diego Palomino Avellaneda ${ }^{1}$
}

\begin{abstract}
Resumen
El objetivo del presente trabajo de investigación fue evaluar la calidad del agua en el río Mashcón al determinar características fisicoquímicas y microbiológicas, y relacionarlas con los Estándares de Calidad Ambiental ECA's para cuerpos de agua. Para ello, se seleccionaron cinco estaciones de muestreo (E1, E2, E3, E4 y E5) en las cuales se tomaron muestras de agua para su análisis físico, químico y microbiológico; de acuerdo a procedimientos estandarizados. Los parámetros de calidad del agua en las cinco estaciones de muestreo indican una notoria variación en cuanto a variables relacionadas a la cantidad de materia orgánica presente en el agua como el DBO5 y DQO; como también en los coliformes totales. Estos parámetros son asociados a perturbación de origen antrópico, lo que guarda relación con la proximidad de las estaciones de muestreo con asentamientos humanos. Al ser comparados con los estándares de calidad ambiental ECA's para agua la estación E1 sobrepasa notoriamente los $\mathrm{ECA}^{\prime}$ s para $\mathrm{DBO}_{5}$, DQO y coliformes totales. Seguida de la estación E2. Lo que evidencia un fuerte grado de perturbación en el cuerpo de agua, por lo que se concluye que la calidad del agua es deficiente en el río Mashcón, en las estaciones de muestreo próximas a la zona urbana.
\end{abstract}

Palabras clave: Calidad; ECA's; contaminación; Cajamarca; río Mashcón.

\begin{abstract}
The objective of this research paper is to evaluate the water quality of the Mashcón River by determining physicochemical and microbiological characteristics, and relating them to the Environmental Quality Standards (ECA) for the water bodies. For this purpose, five sampling stations were selected (E1, E2, E3, E4 and E5) out of which samples for physical, chemical and microbiological analysis were taken in accordance with the standardized procedures. The water quality parameters in the five sampling stations indicated noticeable trends in terms of variables related to the quantity of organic matter present in the water such as $\mathrm{BOD}_{5}$ and $\mathrm{COD}$, as well as in total coliforms. These parameters were associated with the anthropogenic impact, related to the proximity of sampling stations with human settlements. When it comes to the comparison of station E1 to the environmental quality standards for water (ECA), it clearly exceeded the $\mathrm{ECA}$ for $\mathrm{BOD}_{5}, \mathrm{COD}$ and the total coliforms. The same was observed in station E2. The analysis performed show a great degree of disturbance in the examined water body, which concludes that water quality is poor in the Mashcón River, at sampling stations close to the urban area.
\end{abstract}

Keywords: Quality; ECA's; pollution; Cajamarca; river Mashcón.

\section{Introducción}

El agua es el recurso primordial para la vida y el desarrollo de las actividades del hombre, considerándose un elemento vital del planeta, siendo el medio por el cual la vida evoluciona y en el que la vida existe, al mantener la vida terrestre y proveer de agua al $90 \%$ de la población mundial (FAO, 1992; Cabildo \& Cornago, 2004). Aun así la disponibilidad de este recurso es limitada, siendo las principales fuentes aprovechables por el ser humano los ríos, lagos y aguas subterráneas; por lo que mantener la calidad de las fuentes de agua es de vital importancia para todos. Sin embargo se hace un uso inadecuado de este recurso. El desarrollo de las actividades del hombre quienes disponen del recurso agua, muchas veces de manera inadecuada, generan impactos negativos que alteran la calidad del agua al incorporar diversos contaminantes; estos contaminantes comprenden organismos patógenos, materia orgánica, sólidos, nutrientes, sustancias tóxicas, elementos traza, detergentes, sustancias radiactivas, entre otros (Harrison, 2003; Manahan, 2007). En la Tabla 1 se presentan algunos contaminantes del agua y su impacto. Los contaminantes pueden llegar desde fuentes diversas e intermitentes. Las labores agrícolas que influyen notoriamente en los niveles de nitrógeno y fósforo. Las aguas negras y efluentes industriales que tienen alto contenido orgánico y la escorrentía urbana (Henry y Heinke, 1999; Harrison, 2003; Manahan, 2007). Asimismo la composición de las aguas que se introducen en ríos o lagos puede verse alterada por diversas causas; por ejemplo el agua de lluvia al entrar en contacto con gases industriales, procedentes de fuentes cercanas o distantes al ser arrastrados por el viento, y caer en el área de captación. Por procesos de escorrentía, infiltración o el drenaje natural en una cuenca (Henry \& Heinke, 1999). 
Tabla 1. Tipos generales de contaminantes del agua

\begin{tabular}{cc}
\hline Tipo de contaminante & Impacto \\
\hline Materia orgánica & Calidad del agua, niveles de oxigeno \\
Elementos traza & Salud, biota acuática, toxicidad \\
Contaminantes inorgánicos & Toxicidad, biota acuática \\
Nutrientes & Eutrofización \\
Sustancias que alteran el pH & Calidad del agua, vida acuática \\
Patógenos & Efectos en la salud \\
Detergentes & Eutrofización, biota acuática \\
Metales pesados & Salud, biota acuática, toxicidad \\
Medicamentos & Calidad del agua, vida acuática \\
Plaguicidas & Toxicidad, biota acuática \\
Residuos de petróleo & Vida acuática \\
\hline Fuente: Manahan (2007). &
\end{tabular}

Uno de los ecosistemas acuáticos más afectados son los ríos, pues desde el asentamiento de las primeras grandes ciudades, estos se convirtieron en las principales áreas de descarga de los residuos y efluentes generados en dichas ciudades, conllevando un severo impacto en este sistema, que con el tiempo trajo una serie de complicaciones para el hombre, por lo que asegurar la calidad de los mismos se convirtió en una prioridad de la sociedad. Pero en la actualidad se sigue observando como en diferentes lugares, la falta de educación ambiental o simplemente el accionar inadecuado del hombre continúan afectando la calidad del agua.

Ahora se sabe que es de vital importancia mantener la calidad del agua de los sistemas acuáticos, pero, ¿Qué entendemos por calidad del agua? Para muchos el concepto les resulte difícil de entender y hay quienes lo van a llevar a sus determinados campos de acción, al entender la calidad del agua de acuerdo a sus intereses, lo cual no es del todo incorrecto puesto que el concepto de calidad de agua va a depender del punto de vista que se tome. Como un idea general "La calidad del agua se refiere a las condiciones en que se encuentra el agua respecto a características físicas, químicas y biológicas, en su estado natural o después de ser alteradas por el accionar humano" Monroy (2011). Pero también el concepto aplica en relación al uso que se va a hacer del recurso, por lo que el concepto de calidad varía de acuerdo al tipo de uso que se vaya a dar. Los requisitos para la calidad del agua se establecen de acuerdo con el uso al que se destina la misma, ajustándose a estándares que fija el usuario (Harrison, 2003). Por ello, que se dijo que el concepto va a tomar distintas interpretaciones dependiendo de quién se lo plantee, más no podemos desligar la idea de la importancia que representa mantener la calidad del agua para el hombre, puesto que tiene una relación directa con las actividades que este realiza. La alteración de la calidad del agua va a conllevar un impacto directo al hombre; siendo varios los problemas derivados de la contaminación de cuerpos de agua, como son: la reducción del suministro de agua dulce, riesgos en la salud, la inutilización del uso del agua para diversos usos, el impacto negativo sobre la vida acuática (e industria pesquera) y disminuyan el valor estético y de recreación, son solo algunos de los efectos asociados a calidad de agua FAO (1992) y Cardona (2003).
De lo descrito anteriormente, queda claro, que es de suma importancia asegurar la calidad de los cuerpos de agua. Para lo cual se debe evaluar diferentes características, componentes y/o parámetros dentro de este sistema, para realizar un diagnóstico adecuado de la calidad ambiental del agua. Por ello, el objetivo del presente trabajo de investigación fue evaluar la calidad del agua en el río Mashcón al determinar las características fisicoquímicas y microbiológicas de esta y relacionarlas con los Estándares de Calidad Ambiental ECA's para cuerpos de agua.

\section{Área de estudio}

Se realizó un recorrido por el río Mashcón, iniciando a la altura de la estación hidrológica Mashcón ubicada en el puente que une la vía Cajamarca-Baños en el distrito Cajamarca, hasta la unión de los ríos Grande y Porcón que forman el río Mashcón. La selección de las estaciones de muestreo se realizó en función de los problemas ambientales que se pueden asociar a la alteración de la calidad del agua en el río Mashcón, y al grado de accesibilidad en cada estación. Determinándose cinco estaciones de muestreo, que incluyó la selección de un punto testigo. A fin de comparar la variación de los parámetros del área influenciada notoriamente por actividades antrópicas y un área con una perturbación menor; la ubicación de las estaciones de muestreo se presentan en la Tabla 2, siendo la E5 la estación testigo. Así mismo en la Figura 1 se muestra una vista satelital de las estaciones de muestreo en el río Mashcón.

Tabla 2. Ubicación de las estaciones de muestreo en el río Mashcón

\begin{tabular}{|c|c|c|}
\hline Estación & Altitud(m) & Coordenadas \\
\hline \multirow{2}{*}{ E1 } & \multirow{2}{*}{2689} & $7^{\circ} 8^{\prime} 37.92$ ' $\mathrm{S}$ \\
\hline & & $78^{\circ} 29^{\prime} 44.67^{\prime \prime} \mathrm{W}$ \\
\hline \multirow{2}{*}{ E2 } & \multirow{2}{*}{2712} & $7^{\circ} 8$ '33.34 ' $\mathrm{S}$ \\
\hline & & $78^{\circ} 30^{\prime} 51.21^{\prime} ' \mathrm{~W}$ \\
\hline \multirow{2}{*}{ E3 } & \multirow{2}{*}{2725} & $7^{\circ} 8^{\prime} 28.74^{\prime \prime} \mathrm{S}$ \\
\hline & & $78^{\circ} 31^{\prime} 25.40^{\prime \prime} \mathrm{W}$ \\
\hline \multirow{2}{*}{ E4 } & \multirow{2}{*}{2753} & $7^{\circ} 7^{\prime} 32.72^{\prime} \mathrm{S}$ \\
\hline & & $78^{\circ} 31^{\prime} 21.40^{\prime} \mathrm{W}$ \\
\hline \multirow{2}{*}{ E5 } & \multirow{2}{*}{2781} & $7^{\circ} 6^{\prime} 54.95$ ' $\mathrm{S}$ \\
\hline & & $78^{\circ} 31^{\prime} 38.45^{\prime} \mathrm{W}$ \\
\hline
\end{tabular}

En cada estación de muestreo se registraron datos complementarios, los que están descritos en la Tabla 3 y permitirán una mejor interpretación de los resultados obtenidos. Durante el recorrido realizado se observó una serie de problemas ambientales resultado del inadecuado accionar por parte de las personas que, incorporan diversos contaminantes al río, entre ellas es el lavado de motos y autos, y arrojo de residuos sólidos, esto último se puede apreciar en toda la rivera del río que colinda con el área urbana. Estos residuos sólidos incluyen: residuos de construcción, residuos orgánicos, bolsas plásticas, papel, cartón, vidrio, fierros, artículos eléctricos, entre otros. 
Además de la descarga de efluentes, aguas residuales de un lavado de autos y aguas servidas, y también se observó extracción de materiales para ser empleados como agregados de construcción.

\section{Material y métodos}

Parámetros de calidad del agua.

Los parámetros de calidad del agua a evaluar estuvieron en relación a lo establecido en el D.S. N 004-2017-MINAM, el cual establece parámetros de acuerdo al uso que se da al cuerpo del agua, para el presente trabajo corresponde a la categoría 3: riego de vegetales y bebida de animales. Que se divide en subcategoría D1 Riego de vegetales y D2 Bebida de animales. El muestreo se realizó en la estación relativamente seca tomándose una muestra sin repetición para cada parámetro en las cinco estaciones, las cuales fueron llevadas al Laboratorio Regional del Agua para el análisis correspondiente.

\section{$\underline{\text { Recogida de datos complementarios }}$}

Se tuvo en cuenta la presencia de flora ribereña, actividades antrópicas, entre otros, de acuerdo al Anexo 1. A demás se registró el caudal $\left(\mathrm{m}^{3} / \mathrm{s}\right)$ del tramo de río en el que se realizó el muestreo, empleando el método de flotadores (Anexo 2).

\section{Resultados y discusión}

Parámetros físico-químicos y microbiológicos de calidad del agua

En cada estación de muestreo se registraron parámetros in situ como el $\mathrm{pH}$, temperatura, conductividad eléctrica, Sólidos totales disueltos (TDS) y salinidad; y se recolectaron muestras de agua que fueron enviadas al Laboratorio Regional del Agua - Gobierno Regional de Cajamarca para su análisis. Los resultados de los parámetros físicos y químicos se muestran en la Tabla 4.

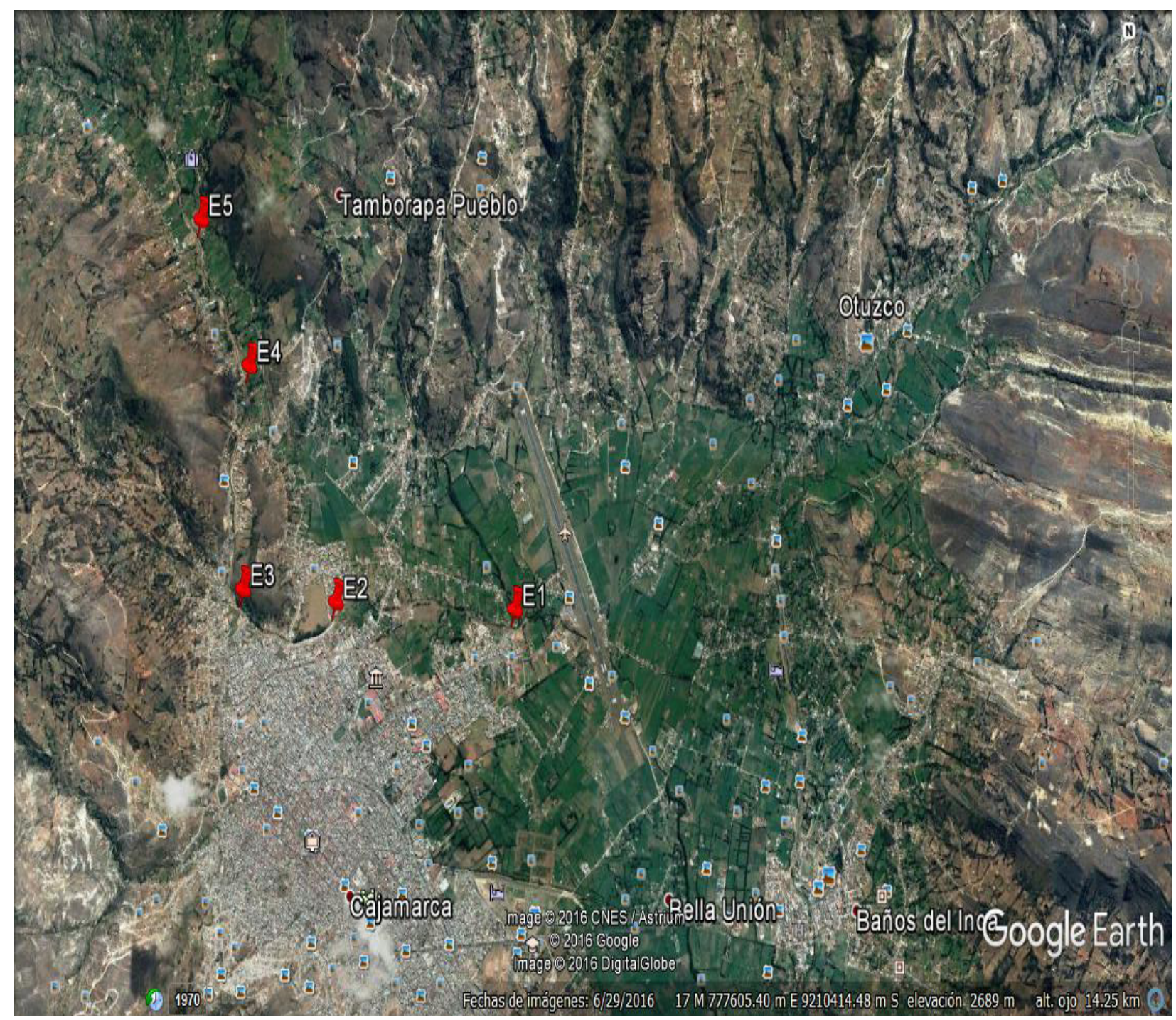

Figura 1. Vista satelital de las estaciones de muestreo en el río Mashcón 
Tabla 3. Datos complementarios registrados en cada estación de muestreo

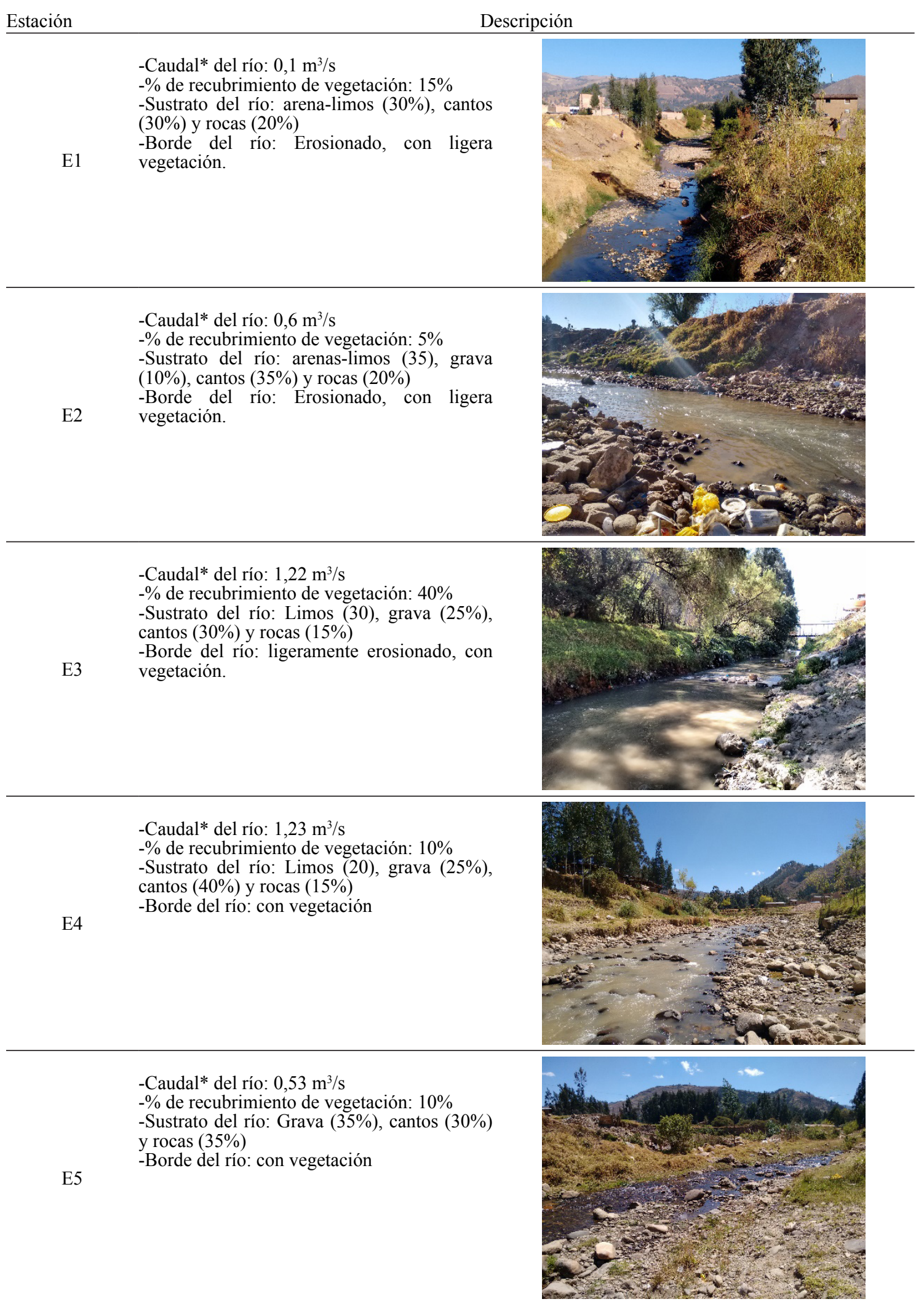

*Caudal: Se calculó empleado el método por flotadores 
Tabla 4. Parámetros de calidad de agua registrados por estación de muestreo en el río Mashcón

\begin{tabular}{|c|c|c|c|c|c|c|c|}
\hline \multirow{2}{*}{ Parámetro } & \multirow{2}{*}{ Unidad } & \multicolumn{5}{|c|}{ Estación } & \multirow{2}{*}{$-\mathrm{ECA}^{\prime}$} \\
\hline & & E1 & E2 & E3 & E4 & E5 & \\
\hline Potencial de hidrógeno $(\mathrm{pH})$ & $\mathrm{pH}$ & 7,10 & 7,08 & 7,25 & 7,30 & 7,40 & $6,5-8,4$ \\
\hline Conductividad eléctrica (CE) & $\mu \mathrm{S} / \mathrm{cm}$ & 746 & 446 & 424 & 423 & 419 & 2500 \\
\hline Sólidos totales disueltos (TDS) & $\mathrm{mg} / \mathrm{L}$ & 520 & 310 & 300 & 300 & 290 & - \\
\hline Temperatura $(\mathrm{T})$ & ${ }^{\circ} \mathrm{C}$ & 19,5 & 17,5 & 16,8 & 15,2 & 14,0 & $\Delta 3$ \\
\hline Salinidad & $\mathrm{mg} / \mathrm{L}$ & 360 & 210 & 190 & 190 & 200 & - \\
\hline Cloruro $\left(\mathrm{Cl}^{-}\right)$ & $\mathrm{mg} / \mathrm{L}$ & 6,94 & 1,30 & 1,16 & 1,09 & 0,92 & 500 \\
\hline Nitrato $\left(\mathrm{NO}_{3}^{-}\right)$ & $\mathrm{mg} / \mathrm{L}$ & 0,41 & 1,59 & 1,15 & 0,91 & 0,96 & 100 \\
\hline Fosfato $\left(\mathrm{PO}_{4}^{3-}\right)$ & $\mathrm{mg} / \mathrm{L}$ & 1,25 & 0,06 & 0,06 & 0,06 & 0,06 & - \\
\hline Sólidos suspendidos totales (SST) & $\mathrm{mg} / \mathrm{L}$ & 101,0 & 120,0 & 31,8 & 948,0 & 3,2 & - \\
\hline Oxígeno disuelto & $\mathrm{mg} / \mathrm{L}$ & 5,69 & 5,79 & 6,30 & 6,59 & 6,93 & $\geq 4$ \\
\hline Demanda bioquímica de oxigeno $\left(\mathrm{DBO}_{5}\right)$ & $\mathrm{mgO}_{2} / \mathrm{L}$ & 94,0 & 7,7 & 2,0 & 9,4 & 2,0 & 15 \\
\hline Demanda química de oxigeno (DQO) & $\mathrm{mgO}_{2} / \mathrm{L}$ & 250 & 26 & 8 & 66 & 8 & 40 \\
\hline Coliformes totales & $\mathrm{NMP} / 100 \mathrm{~mL}$ & $92 * 10 \square$ & $14 * 10 \square$ & 7000 & 2000 & 1400 & - \\
\hline
\end{tabular}

En cuanto a los valores de Temperatura estos fueron disminuyendo desde la estación uno a la estación cinco, (Figura 3); lo mismo que los valores de conductividad eléctrica Figura 4 y sólidos totales disueltos (TDS) Figura 5. En cuanto al $\mathrm{pH}$ se observa que este registro los menores valores
Entre los resultados más resaltantes podemos describir los siguientes: La estación E5, fue la estación testigo registró valores de $6,93 \mathrm{mg} / \mathrm{L}$ para oxígeno disuelto, los valores de $\mathrm{DBO}_{5}$ y DQO fueron de $2 \mathrm{mgO}_{2} / \mathrm{L} \mathrm{y} 8 \mathrm{mgO}_{2} / \mathrm{L}$ respectivamente, en cuanto a Coliformes Totales se registró un valor de $1400 \mathrm{NMP} / 100 \mathrm{~mL}$. En la estación E4 se obtuvieron valores de $6,3 \mathrm{mg} / \mathrm{L}$ para oxígeno disuelto, los valores de $\mathrm{DBO}_{5}$ y DQO fueron de $2 \mathrm{mgO}_{2} / \mathrm{L}$ y 8 $\mathrm{mgO}_{2} / \mathrm{L}$ respectivamente, en cuanto a Coliformes Totales se registró un valor de $2000 \mathrm{NMP} / 100 \mathrm{~mL}$. La estación E3, registró valores de $6,59 \mathrm{mg} / \mathrm{L}$ para oxígeno disuelto, los valores de $\mathrm{DBO}_{5}$ y $\mathrm{DQO}$ fueron de $9,4 \mathrm{mgO}_{2} / \mathrm{L}$ y $66 \mathrm{mgO}_{2} / \mathrm{L}$ respectivamente, en cuanto a Coliformes Totales se registró un valor de $7000 \mathrm{NMP} / 100 \mathrm{~mL}$. En los siguientes dos estaciones es en donde se pudo observar los mayores problemas ambientales que puedan afectar la calidad del agua. La estación E2 registró valores de 5,79 $\mathrm{mg} / \mathrm{L}$ para oxígeno disuelto, los valores de $\mathrm{DBO}_{5}$ y DQO fueron de $7,7 \mathrm{mgO} 2 / \mathrm{L}$ y $26 \mathrm{mgO}_{2} / \mathrm{L}$ respectivamente, en cuanto a Coliformes Totales se registró un valor de 14x10 $\square \mathrm{NMP} / 100 \mathrm{~mL}$, se pudo observar residuos sólidos en las orillas y materia orgánica en descomposición. En la estación E1 se obtuvieron valores de 5,69 mg/L para oxígeno disuelto siendo el valor más bajo registrado, los valores de $\mathrm{DBO}_{5}$ y DQO fueron de $94 \mathrm{mgO}_{2} / \mathrm{L}$ y 250 $\mathrm{mgO}_{2} / \mathrm{L}$ respectivamente, en cuanto a Coliformes Totales se registró un valor de $92 \times 10 \square \mathrm{NMP} / 100 \mathrm{~mL}$, se pudo observar residuos sólidos en las orillas, materia orgánica en descomposición, descarga de efluentes, presencia de animales mayores (vacunos).

Los sólidos suspendidos totales (SST) mostraron una variación abrupta en las estaciones, específicamente en la estación E4 que registró el valor más elevado en comparación con las demás estaciones (Figura 2), lo que explica la regular turbidez observada durante el muestreo. Esto posiblemente se deba a que aguas arriba se realizan trabajos con maquinaria pesada en el lecho del río, lo que ocasione un aumento de los sólidos en suspensión en el agua. en las estaciones de muestreo E1 y E2 (Figura 6). Los aniones: cloruros $\left(\mathrm{Cl}^{-}\right)$, nitratos $\left(\mathrm{NO}_{3}^{-}\right)$y fosfatos $\left(\mathrm{PO}_{4}^{3-}\right)$ mostraron valores relativamente homogéneos, a excepción de la estación E1 que muestra valores más elevados para cloruros y fosfatos (Figura 7).

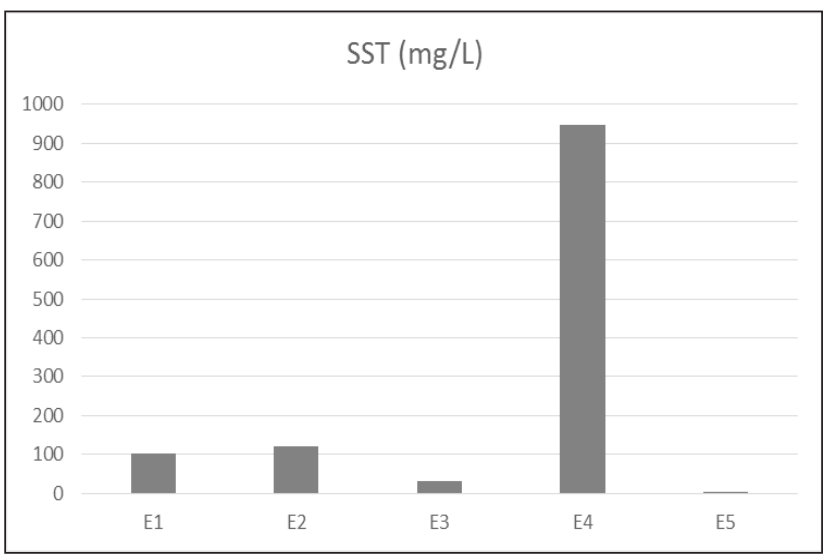

Figura 2. Gráfica de sólidos suspendidos totales por estación de muestreo

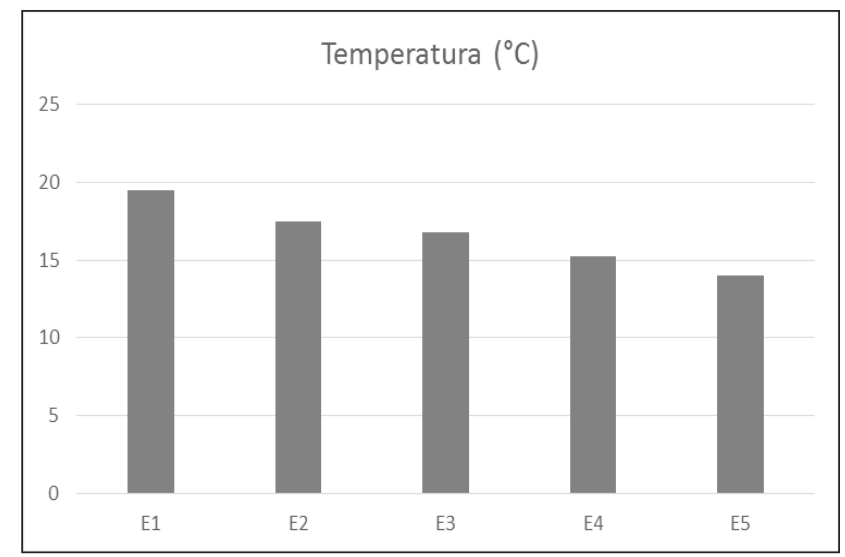

Figura 3. Gráfica de la Temperatura por estación de muestreo 


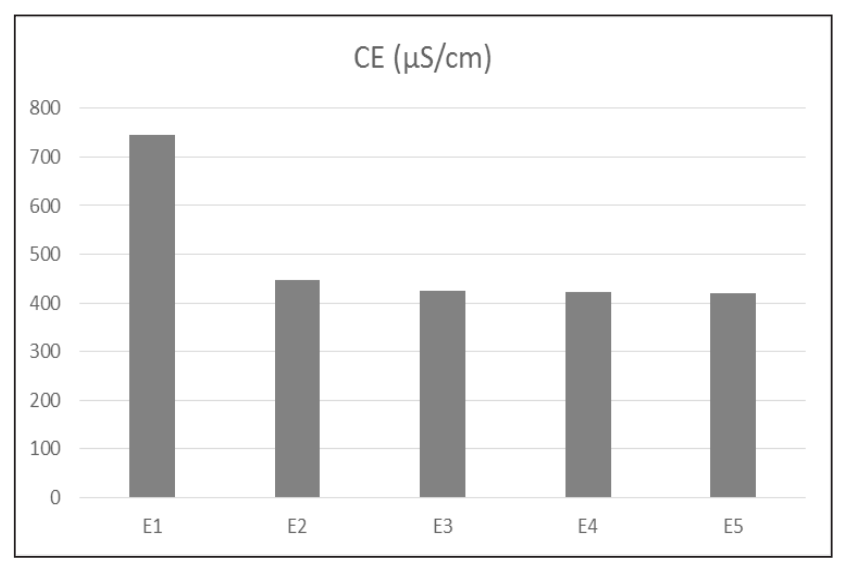

Figura 4. Gráfica de conductividad eléctrica por estación de muestreo

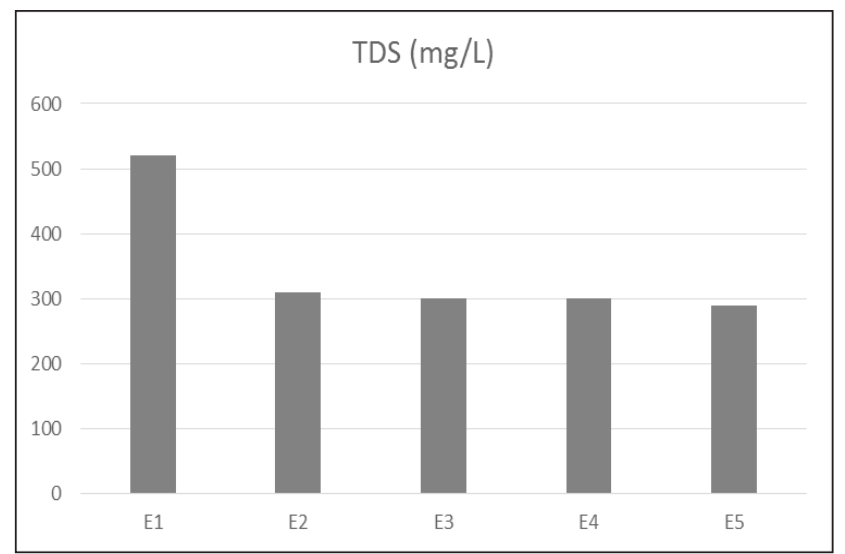

Figura 5. Gráfica de sólidos totales disueltos por estación de muestreo

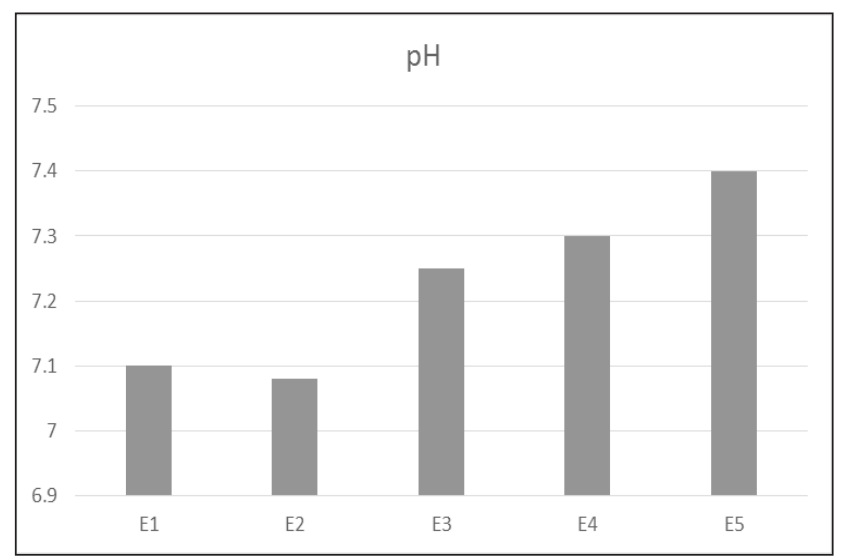

Figura 6. Gráfica de $\mathrm{pH}$ por estación de muestreo

Si relacionamos las cinco estaciones de muestreo podemos apreciar como hay una variación entre el testigo y las cuatro estaciones, principalmente en cuanto a variables asociadas a la cantidad de materia orgánica presente en el agua como el $\mathrm{DBO}_{5}$ y DQO, Figura 8 y 9 respectivamente. En el caso de los coliformes totales, se observa una notoria variación, puesto que es mucho.

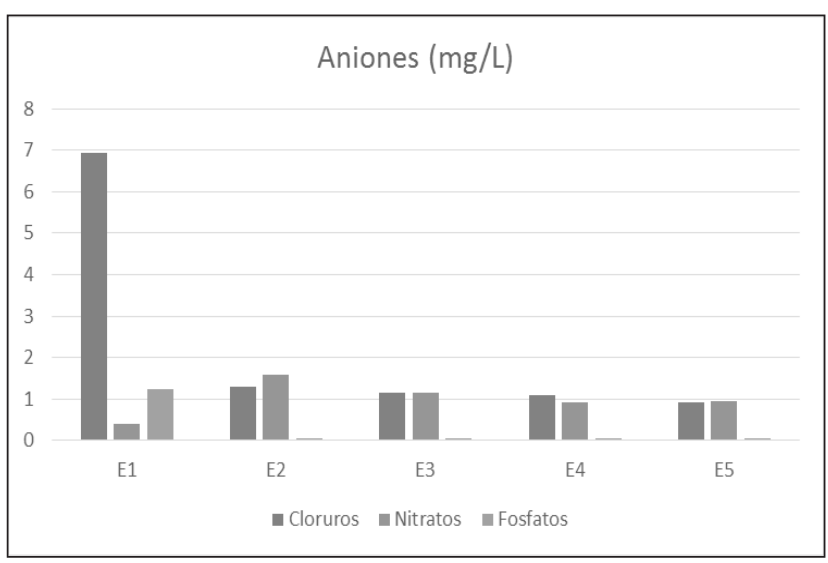

Figura 7. Gráfica de Aniones por estación de muestreo

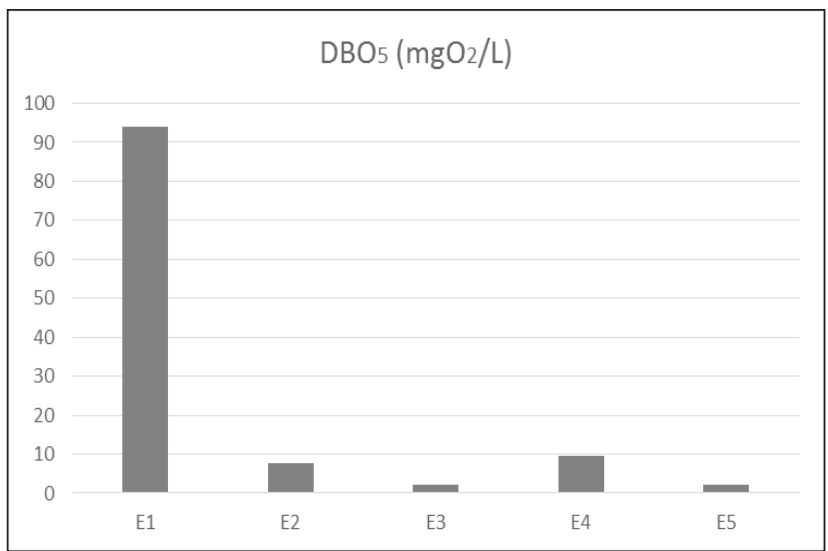

Figura 8. Gráfica de la demanda bioquímica de oxígeno por estación de muestreo

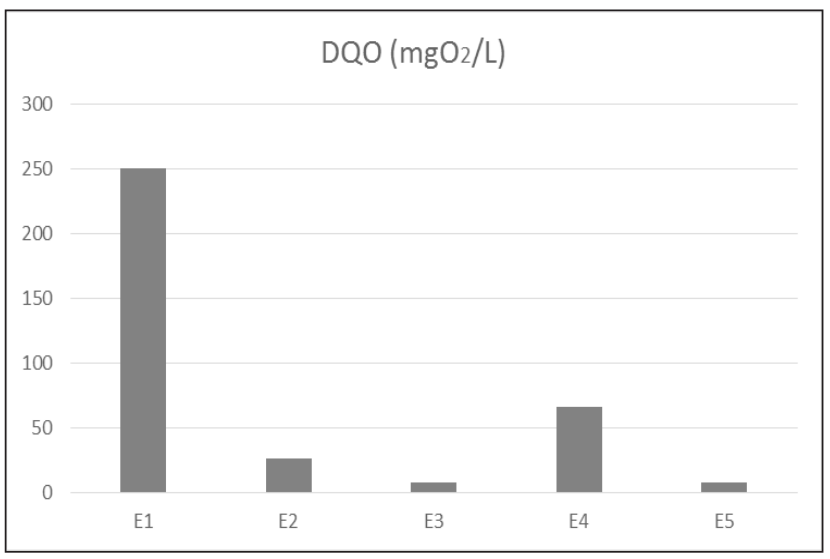

Figura 9. Gráfica de la demanda química de oxígeno por estación de muestreo

Si relacionamos los datos abióticos según el diagrama de Draftsman plot (Figura 10), podemos encontrar una correlación positiva del parámetro temperatura con la conductividad eléctrica (CE), sólidos totales disueltos (TDS), demanda química de oxigeno (DQO) y demanda bioquímica de oxigeno $\left(\mathrm{DBO}_{5}\right)$; lo mismo ocurre con los sólidos totales y la conductividad mostrando una relación 
también directamente proporcional. En tanto que hay una correlación negativa entre la temperatura y el oxígeno disuelto, así como también con el pH.

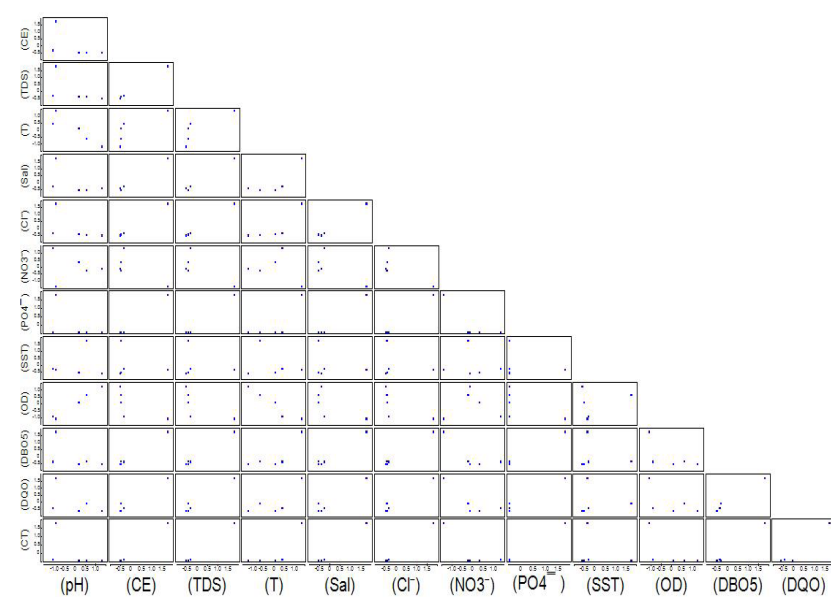

Figura 10. Diagrama de Draftsman plot

El río Mashcón se ve influenciado por el desarrollo urbano y otras actividades principalmente agricultura y ganadería; teniendo una influencia variada en las estaciones seleccionadas, así tenemos que las estaciones E1, E2 y E3 son las que tienen una mayor presión por las actividades del ser humano, al estar próximas a zonas urbanas. Aunque los asentamientos humanos mantienen su influencia en las estaciones E4 y E5, esta va disminuyendo, pero las actividades agropecuarias son las que toman mayor notoriedad.

En cuanto a la descripción de cada una de las estaciones de muestreo, se registró el caudal en las cinco estaciones, variando este entre $0,10 \mathrm{~m}^{3} / \mathrm{s}$ a $1,23 \mathrm{~m}^{3} / \mathrm{s}$; siendo la estación E1 la que registró el menor valor. Esto debido a que el muestreo se realizó en la época de estiaje, pero también puede ser influenciado por modificaciones en el lecho del río aguas arriba.

El tipo de sustrato del río, presencia de vegetación ribereña y área boscosa, y la estabilidad de los bordes variaron en cada estación de muestreo; y según lo señalado por Oyanedel et al. (2008) estas características tienen una gran importancia ecológica, al ser factores controladores de los patrones de distribución de comunidades de organismos bentónicos. Meza et al. (2012) describe que los fondos arenosos albergan pocas especies, con pocos individuos por especie, los fondos pedregosos suelen ser más ricos, en especial cuando las rocas son grandes y en presencia de vegetación la fauna tiende a ser mucho más diversa. Esto conlleva a que en este tipo de ambientes haya una mayor diversidad de especies.

Los parámetros evaluados en las estaciones de muestreo del río Mashcón presentaron ciertas variaciones, y en algunos casos determinados parámetros mostraron una notoria variación en relación a las demás estaciones. Estos parámetros son analizados según el Decreto Supremo N ${ }^{\circ}$ 004-2017-MINAM que establece el Estándar Nacional de
Calidad Ambiental (ECA) para Aguas, correspondiéndole la categoría III: Riego de vegetales y bebida de animales. Que a su vez se divide en: Subcategoría D1: Riego de vegetales; la cual se subdivide en agua para riego no restringido y agua para riego restringido. Y Subcategoría D2: Bebida de animales.

$\mathrm{El} \mathrm{pH}$ en las estaciones de muestreo es relativamente similar con tendencia a tomar un carácter básico ya que los valores están entre 7,08 y 7,40. Entre las cinco estaciones de muestreo los valores de las estaciones E1 y E2 son los más bajos. El valor del ECA para aguas de categoría III es de $6,5-8,4$ lo que indica que estaría dentro del rango.

La conductividad eléctrica (CE) del agua del río Mashcón es baja, entre 419-746 uS/cm, esto en comparación con lo establecido en el ECA para aguas de categoría III $(2500 \mathrm{uS} / \mathrm{cm})$. La estación de muestreo E1 es la que presenta la mayor conductividad entre las estaciones, siendo esta estación la que presenta los mayores problemas ambientales como descarga de efluentes y residuos sólidos en la ribera y el cauce. Al respecto Arroyo (2007) señala que la conductividad puede ser considerada una buena medida de contaminación al estar directamente relacionada a la concentración de iones en el agua. Probablemente debido a la relativa mayor concentración de asentamientos humanos existentes alrededor de dichas fuentes de agua, las cuales pueden encontrarse vertiendo desechos orgánicos, fertilizantes o pesticidas provenientes de actividades domésticas, agrícolas o ganaderas.

Los valores de sólidos totales disueltos (TDS) están entre 290 y $520 \mathrm{mg} / \mathrm{L}$, valores bajos si los comparamos con un agua que puede ser potabilizada por desinfección ya que está por debajo de los $(1000 \mathrm{mg} / \mathrm{L})$.

Los aniones (cloruros, fosfatos y nitratos) registran todos valores inferiores en relación con el ECA para aguas de categoría III. La determinación de fosfatos y nitratos es importante ya que permite detectar problemas de eutrofización de los cuerpos de agua (Rivera et al., 2008 en Custodio \& Chaname, 2016).

El oxígeno disuelto (OD) según el ECA para aguas de categoría III, estas aguas aceptan un valor mínimo para este parámetro de $4 \mathrm{mg} / \mathrm{L}$, en la estación cinco el valor es el más adecuado puesto que refleja un agua bien oxigenada y que no generaría problemas a la biota que se desarrolla en este ambiente, en tanto que, en las estaciones E1 y E2 se observa una disminución en los valores de oxígeno disuelto. Custodio \& Chaname (2016) precisan que la cantidad de oxígeno depende de las características del cauce, la turbulencia del agua y los procesos químicos y biológicos. Siendo este gas, conjuntamente con la temperatura, determinante en la riqueza y los patrones de distribución de las familias de macroinvertebrados bentónicos

La demanda bioquímica de oxigeno $\left(\mathrm{DBO}_{5}\right)$ según el ECA para aguas de categoría III no debe ser mayor a 15 mg/L. En las estaciones E2, E3, E4 y E5 los valores no sobrepasan el valor máximo establecido, caso contrario sucede en la estación E1 que sobrepasa notoriamente este valor al presentar $94 \mathrm{mg} / \mathrm{L}$. 
En cuanto a la demanda química de oxigeno (DQO) el ECA para aguas de categoría III establece que no debe superar los $40 \mathrm{mg} / \mathrm{L}$. y al igual que la $\mathrm{DBO}_{5}$ es en la estación E1 donde se observa una gran variación (250 $\mathrm{mg} / \mathrm{L}$ ) en cuanto al valor máximo aceptado.

$\mathrm{La} \mathrm{DBO}_{5}$ y la DQO miden en términos sencillos la cantidad de materia orgánica presente en el cuerpo de agua, teniendo una alta influencia en el ecosistema acuático. Custodio \& Chaname (2016) señalan que las actividades sin criterios ambientales está dando lugar al vertido de cargas excesivas de contaminantes orgánicos, lo que aumenta la materia orgánica disponible en el agua, reduciendo cada vez más la capacidad de los ecosistemas acuáticos de eliminar estos desechos, lo que pone en peligro la sostenibilidad del suministro de alimentos y la biodiversidad.

En relación a los coliformes totales hay una notoria diferencia en el valor máximo aceptado entre las subcategorías establecidas: para riego de vegetales acepta un valor máximo de $1000 \mathrm{NMP} / 100 \mathrm{~mL}$ y para bebidas de animales $5000 \mathrm{NMP} / 100 \mathrm{~mL}$. En las cinco estaciones de muestreo, son la E1 y E2 las que superan ampliamente estos valores, en tanto que las estaciones E3, E4 y E5 muestran valores superiores solo para la subcategoría riego de vegetales. Lo que evidenciaría el vertimiento, en mayor o menor medida, de efluentes de origen pecuario y urbano sin tratamiento previo al curso del río, pues la presencia de estas bacterias en el agua revela contaminación fecal.

Los parámetros físicos, químicos y microbiológicos van a influenciar el ecosistema acuático, modificando el hábitat en el que se desarrollan los organismos. Así lo señalan Rivera et al. (2013) y Gil (2014) indicando que existe una correlación entre los parámetros fisicoquímicos del agua determinados por factores ambientales y la diversidad de las comunidades de organismos bentónicos. Factores como la profundidad, $\mathrm{pH}$, alcalinidad, dureza, iones de calcio, clase de sedimentos, materia orgánica, contaminantes tanto industriales como domésticos, determinan la abundancia relativa de estas comunidades. La alteración de estos parámetros por la presencia de contaminantes que de forma individual o por procesos sinérgicos van a modificar el ecosistema. Galindo et al. (2012), Gil (2014) señalan que en el caso de la materia orgánica, esta al acumularse inicia un proceso de descomposición consumiendo también una buena parte del oxígeno disuelto presente en el agua, lo que se refleja en el incremento de los valores de DBO y otras características fisicoquímicas (como los sólidos en suspensión, la dureza, entre otros), los cuales afectan de manera directa las comunidades de organismos cuyas especies menos tolerantes van a desaparecer. Morelli \& Verdi (2014) señalan que el pH, la conductividad, el oxígeno disuelto y la temperatura, son los parámetros determinantes en la distribución de los organismos acuáticos y a los que estos son más sensibles. Si bien es cierto en el presente estudio estos parámetros no presentaron cambios notorios entre las estaciones y se mantuvieron dentro del máximo valor aceptado según los ECA's para aguas de categoría III; por lo que podemos decir que su influencia fue menor, esto en relación a la $\mathrm{DBO}_{5}$ y la DQO que si mostraron cambios evidentes entre estaciones, superando notoriamente los valores ECA's mínimos establecidos en las dos primeras estaciones de muestreo; las cuales están próximas a la zona urbana. Evidenciando que la mayor presión ejercida sobre el cuerpo de agua está en relación a la proximidad de el área urbana al río. Por lo que es necesario complementar este trabajo con investigaciones enfocadas en caracterizar los contaminantes de mayor impacto, para así plantear medidas de solución a esta problemática ambiental, que no solo afecta al ecosistema, sino también a las personas que sustentan sus actividades en el recurso agua.

\section{Conclusiones}

Los resultados obtenidos en la determinación de parámetros físicos, químicos y microbiológicos indican que la estación E1 sobrepasa notoriamente los estándares de calidad ambiental para $\mathrm{DBO}_{5}$, DQO y coliformes totales. Seguida de la estación E2. Lo que evidencia un fuerte grado de perturbación en el cuerpo de agua. Por lo que podemos concluir que hay una deficiente calidad del agua en estos puntos muestreados del río Mashcón, y está asociado a la proximidad del río a la zona urbana.

\section{Agradecimiento}

Un agradecimiento muy sincero al Ing. Gary Christiam Farfán Chilicaus por su inconmensurable aporte profesional y su constante apoyo siempre con gentiliza y con el deseo de ayudar, a quien siempre le estaré agradecido.

\section{Literatura citada}

Arroyo, D. 2007. Evaluación de la calidad de agua de las fuentes hidrográficas del Bosque Protector Río Guajalito (BPRG) a través de la utilización de macroinvertebrados acuáticos, Pichincha, Ecuador. Universidad San Francisco de Quito, Ecuador.

Cabildo, M. \& y Cornago, M. 2004. Bases químicas del medio ambiente. Madrid: Universidad Nacional de Educación a distancia.

Cardona, A. 2003. Calidad y riesgo de contaminación de las aguas superficiales en la microcuenca del Río La Soledad, Valle de Ángeles, Honduras. Tesis. Centro Agronómico Tropical de Investigación y Enseñanza, Costa Rica. Disponible en: http://orton.catie.ac.cr/ repdoc/A0118e/A0118e.pdf

Custodio, M. \& Chaname, F. 2016. Análisis de la biodiversidad de macroinvertebrados bentónicos del río Cunas mediante indicadores ambientales, Junín-Perú. Scientia Agropecuaria, 7 (1): 33 - 44.

DECRETO SUPREMO 014-2017-MINAM. Aprueban Estándares de Calidad Ambiental (ECA) para Agua y establecen Disposiciones Complementarias (Junio 07, 2017)

FAO. 1992. Prevención de la contaminación del agua por 
la agricultura y actividades a fin. Informes sobre temas hídricos. Santiago de Chile. Disponible en: https:// books.google.com.pe/books? $\mathrm{id}=1$ uuxpntvq8C \&dq=FA O. + Prevenci $\% \mathrm{C} 3 \% \mathrm{~B} 3 \mathrm{n}+\mathrm{de}+\mathrm{la}+$ contaminaci $\% \mathrm{C} 3 \% \mathrm{~B} 3$ $\mathrm{n}+\mathrm{del}+$ agua + por + la + agricultura $+\mathrm{y}+$ actividades $+\mathrm{a}+$ fin. $\& \mathrm{hl}=$ es\&source $=$ gbs_navlinks_s

Galindo, L.; Constantino, L.; Benavides, P.; Montoya, E. y Rodríguez, N. 2012. Evaluación de macroinvertebrados acuáticos y calidad de agua en quebradas de fincas cafeteras de Cundinamarca y Santander, Colombia. Revista Cenicafé, 63(1):70-92.

Gil, J. 2014. Determinación de la calidad del agua mediante variables físico químicas, y la comunidad de macroinvertebrados como bioindicadores de calidad del agua en la cuenca del río Garagoa. Universidad de Manizales

Harrison, R. 2003. El Medio Ambiente: Introducción a la química medioambiental y a la contaminación. Zaragoza, España: Acribia.

Henry, J. \& Heinke, G. 1999. Ingeniería ambiental. México D.F.: Prentice Hall.

Manahan, S. 2007. Introducción a la química ambiental. México D.F.: Reverté.

Meza, A.; Rubio, J.; Dias, L \& Walteros, J. 2012. Calidad de agua y composición de macroinvertebrados acuáticos en la subcuenca alta del río Chinchiná. Revista Caldasia, 34(2): 443-456. Disponible en: http:// www.icn.unal.edu.co/

Monroy, N. 2011. Análisis de la situación jurídica actual de los recursos hídricos en la república de Guatemala y la necesidad de crear la ley de aguas y rectoría del recurso hídrico. Tesis de pregrado). Universidad de San Carlos de Guatemala, Guatemala. Recuperado de: http://biblioteca.usac.edu.gt/tesis/04/04_9145.pdf

Morelli, E. \& Verdi, A. 2014. Diversidad de macroinvertebrados acuáticos en cursos de agua dulce con vegetación ribereña nativa de Uruguay. Revista Mexicana de Biodiversidad, 85: 1160-1170.

Oyanedel, A.; Valdovino, C.; Azócar, M.; Moya, C.; Mancilla, G.; Pedreros, P. \& Figueroa, R. 2008. Patrones de distribución espacial de los macroinvertebrados bentónicos de la cuenca del río Aysén (Patagonia chilena). Revista Gayana, 72(2): 241-257.

Rivera, J.; Pinilla, G. \& Rangel, J. 2013. Ensamblaje de macroinvertebrados acuáticos y su relación con las variables físicas y químicas en el humedal de JaboqueColombia. Revista Caldasia, 35(2):389-408. Disponible en: http://www.icn.unal.edu.co/ 
Anexo 1. Descripción de la estación de muestreo

\begin{tabular}{|c|c|c|c|}
\hline \multicolumn{4}{|l|}{ DATOS GENERALES } \\
\hline \multicolumn{2}{|l|}{ Nombre del río: } & & Microcuenca: \\
\hline \multicolumn{2}{|l|}{ Provincia: } & & Fecha y Hora: \\
\hline \multirow{2}{*}{\multicolumn{2}{|c|}{$\begin{array}{l}\text { Responsable: } \\
\text { Clima: }\end{array}$}} & \\
\hline & Equipo: & & \\
\hline \multicolumn{4}{|l|}{ DESCRIPCIÓN DEL RÍO } \\
\hline ¿El agua del río fluye? & Sí: & No: & Caudal: \\
\hline \multirow{3}{*}{$\begin{array}{l}\text { ¿El nivel del agua es } \\
\text { habitual para la época } \\
\text { del año? }\end{array}$} & \multicolumn{2}{|l|}{ Sí: } & \multirow{4}{*}{ Sombra sobre el río: } \\
\hline & \multicolumn{2}{|l|}{ Mayor: } & \\
\hline & \multicolumn{2}{|l|}{ Menor: } & \\
\hline \multirow{4}{*}{$\begin{array}{l}\text { Condición de la orilla del } \\
\text { río }\end{array}$} & \multicolumn{2}{|l|}{ Con vegetación: } & \\
\hline & \multicolumn{2}{|l|}{ Erosionado: } & \multirow{4}{*}{ Sustrato del río: } \\
\hline & \multicolumn{2}{|l|}{ Urbanizado } & \\
\hline & \multicolumn{2}{|l|}{ Otro: } & \\
\hline \multirow{5}{*}{$\begin{array}{l}\text { Uso del suelo en área } \\
\text { circundante }\end{array}$} & \multicolumn{2}{|l|}{ Agropecuario: } & \\
\hline & \multicolumn{2}{|l|}{\begin{tabular}{|l|} 
Industrial: \\
\end{tabular}} & \multirow{2}{*}{ Temperatura: } \\
\hline & \multicolumn{2}{|l|}{ Residencial: } & \\
\hline & \multicolumn{2}{|l|}{\begin{tabular}{|l|} 
Protección: \\
\end{tabular}} & \multirow{2}{*}{$\mathrm{pH}:$} \\
\hline & \multicolumn{2}{|l|}{ Otro: } & \\
\hline \multirow{4}{*}{$\begin{array}{l}\text { Indicios de } \\
\text { contaminación }\end{array}$} & \multicolumn{2}{|l|}{ Residuos sólidos: } & \multirow{2}{*}{ Conductividad } \\
\hline & \multicolumn{2}{|l|}{\begin{tabular}{|l} 
Efluentes industriales: \\
\end{tabular}} & \\
\hline & \multicolumn{2}{|l|}{\begin{tabular}{|l} 
Efluentes domésticos: \\
\end{tabular}} & \multirow{2}{*}{ Oxígeno disuelto: } \\
\hline & \multicolumn{2}{|l|}{ Otro: } & \\
\hline
\end{tabular}

Anexo 2. Control de caudales-Método por flotadores

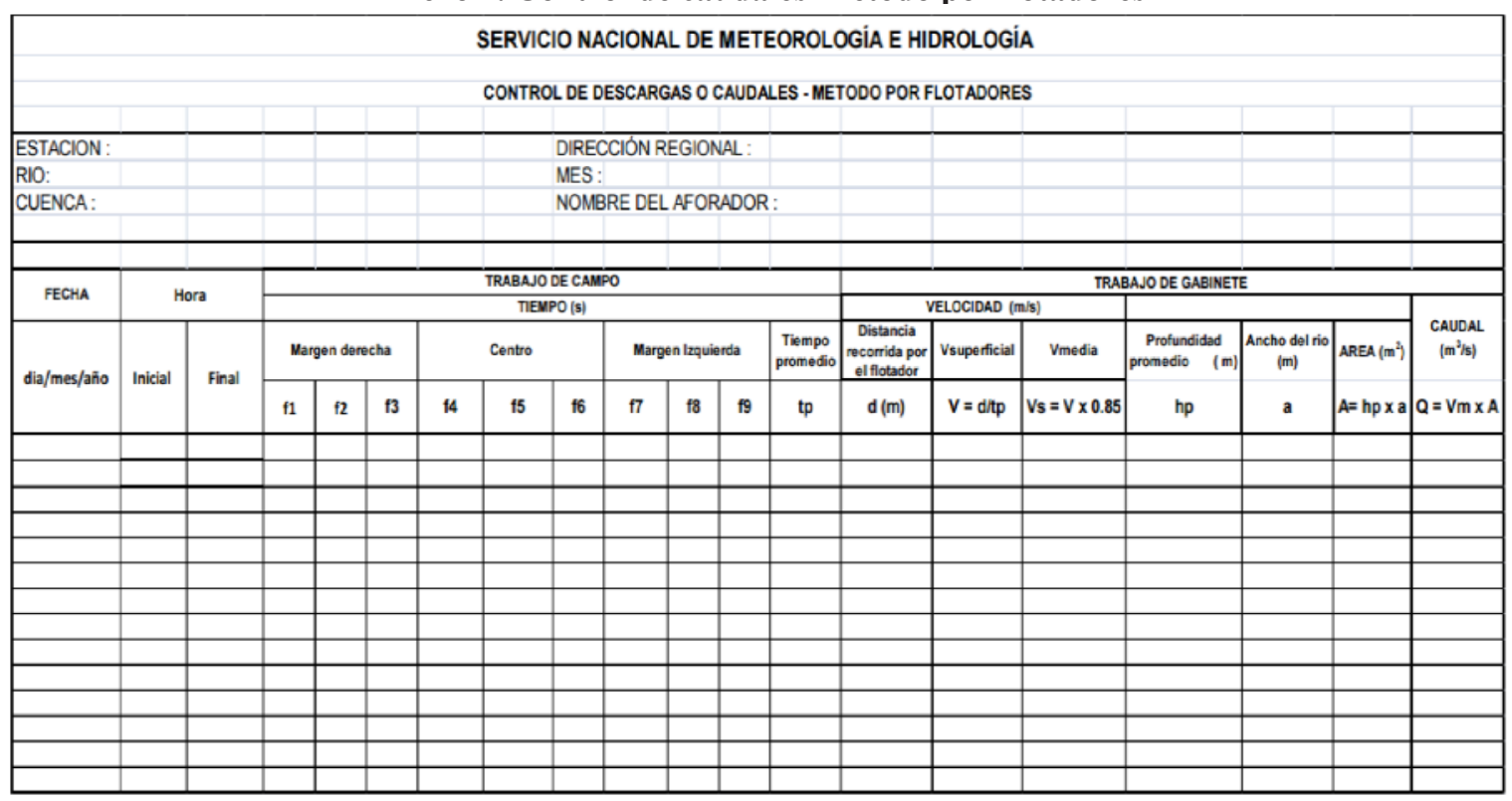

